\title{
Simultaneous High-Speed DualEELS and EDS acquisition at atomic level across the $\mathrm{LaFeO}_{3} / \mathrm{SrTiO}_{3}$ interface
}

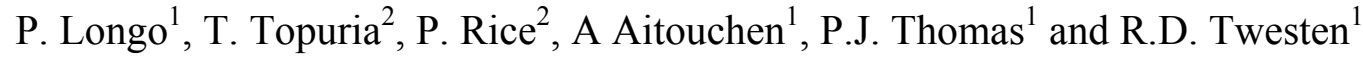 \\ ${ }^{1}$ Gatan Inc. 5794 W Las Positas Blvd, Pleasanton, CA, 94588, USA \\ ${ }^{2}$ IBM Research Division, Almaden Research Center, San Jose, CA, 95120, USA
}

Lanthanum ferrite $\mathrm{LaFeO}_{3}$ (LFO) is an antiferromagnetic perovskite oxide and has been investigated with great interest due to its potential applications in computer hard drives as read heads. LFO films are typically grown on $\mathrm{SrTiO}_{3}$ (STO) substrates due to the well matched lattice constants and the effects of chemistry, morphology and strain at the LFO / STO interface on the magnetic and electrical properties of the system. Hence, the study of the chemistry and elemental distribution at the atomic scale is important in order to understand the properties of these films. EELS and EDS in a STEM microscope have proven to be valuable tools for characterizing such materials.

Here we show an approach where the simultanous high-speed acquisition of EELS and EDS spectra with an atomic scale probe allows the possibility to carry out a full compositional and chemical characterization of the LFO / STO interface. The analysis was carried out using a relatively short exposure time to reduce the effects of beam damage and sample drift which can be more pronounced when the data acquisiton is carried out at atomic level. To achieve efficient and fast joint EELS / EDS data acquisition, we have linked the STEM scan system with the EELS and EDS detectors via a single clock mastered off the EELS detector to ensure all the systems are in exact synchrony. To ensure data fidelity, the native detector applications are used to acquire the data into buffers and the data is transferred to the final application (in this case DigitalMicrograph) in blocks while the CPU is idle.

Data were acquired at IBM San Jose, CA, USA using a probe-corrected, C-FEG STEM operating at 200 kV. A GIF Quantum ER EELS system equipped with DualEELS capability was used for the acquisition of energy-loss data and a large area ( 1 sr) EDS detector was used to acquire the EDS data. EELS data were acquired in DualEELS mode and the low-loss $(0 \mathrm{eV}$ to $500 \mathrm{eV})$ and core-loss (400 eV to $900 \mathrm{eV})$ EELS spectra were acquired nearly simultaneously with $10 \mu \mathrm{s}$ transition time between exposures. The low-loss spectrum provides an accurate energy reference allowing absolute chemical shift measurements. The spectrometer was set up for moderate energy resolution analysis with a dispersion of $0.25 \mathrm{eV} /$ channel yielding a measured energy resolution of $0.75 \mathrm{eV}$ with $500 \mathrm{eV}$ energy range in the spectrum. By looking at the shape of the near edge structure in the EELS spectrum, it is possible to extract information on the oxidation state, coordination state and much more. In addition, optical information present in the low-loss region is also available since the low-loss EELS data were recorded with each core-loss spectrum. EELS data were used to analyze chemical state information and generate elemental maps of the $\mathrm{O}, \mathrm{Ti}, \mathrm{Fe}$ and $\mathrm{La}$. In the EELS spectrum, the main signals for the $\mathrm{Sr}$ are the $\mathrm{M}_{4,5}$ at $130 \mathrm{eV}$ and the $\mathrm{Sr} \mathrm{L}_{2,3}$ at $1940 \mathrm{eV}$. The M-edges have a poor peak-to-background ratio due their low energy while the L-edges are out of the energy range for this experiment $(0 \mathrm{eV}$ to $900 \mathrm{eV})$. The EDS data were acquired simultaneously with the EELS data and can be used to generate a Sr elemental map that can be combined with those obtained by EELS. Since the EDS signal does not provide any chemical information, only composition can be studied for $\mathrm{Sr}$ at the experimental conditions used in this paper.

Figure 1 shows a colorized elemental map of Ti, Fe and La obtained using EELS and Sr using EDS. The color map suggests intermixing between Fe and Ti. Such intermixing can be confirmed and further 
investigated using EELS by looking at the fine structure of the edges across each atomic column. Figures 2a-c show EELS spectra of Ti L, O K and Fe L edges extracted from the selected regions in Figure 1. Changes can be observed in every spectrum although they are notable in the case of the $\mathrm{O}$ and $\mathrm{Fe}$ in Figures $2 \mathrm{~b}$,c with the $\mathrm{Fe}$ showing a chemical shift towards higher energy moving into the $\mathrm{LaFeO}_{3}$. This is a clear indication of a change in the oxidation state. The interface was also analyzed using STEM diffraction where diffraction patterns are taken point by point across the interface. This gives a further insight into the structure across the interface and information about strain and crystal distortion can be revealed.

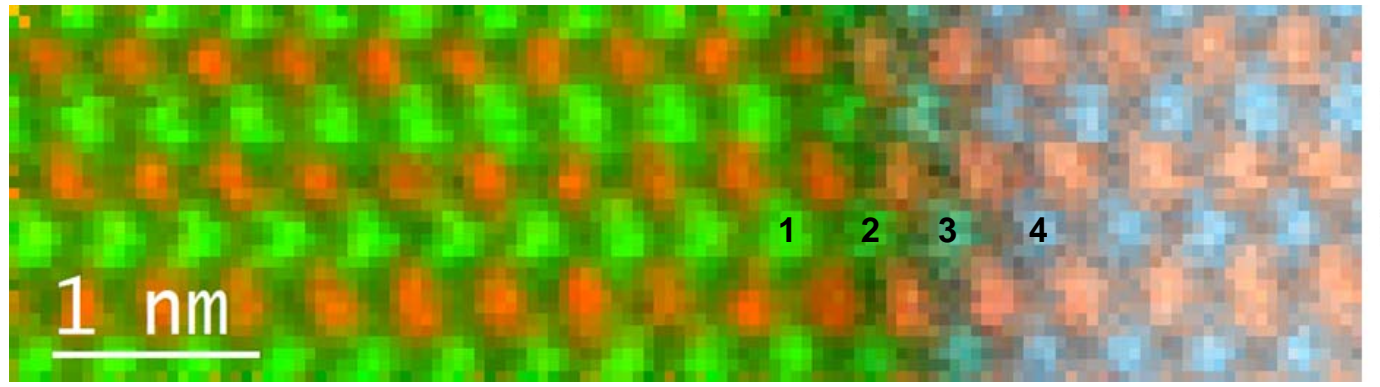

Sr L EDS: red

Ti L EELS: green La M EELS: orange Fe L EELS: light blue

Figure 1: Color maps of Ti $\mathrm{L}$ at $456 \mathrm{eV}$ in green, $\mathrm{La} \mathrm{M}$ at $832 \mathrm{eV}$ in orange and $\mathrm{Fe} \mathrm{L}$ at $708 \mathrm{eV}$ in light blue obtained using EELS and Sr L at $1.81 \mathrm{keV}$ in red obtained using EDS. The numbers $1-4$ correspond to the selected region where the EELS spectra in Figures 2a-d were extracted.
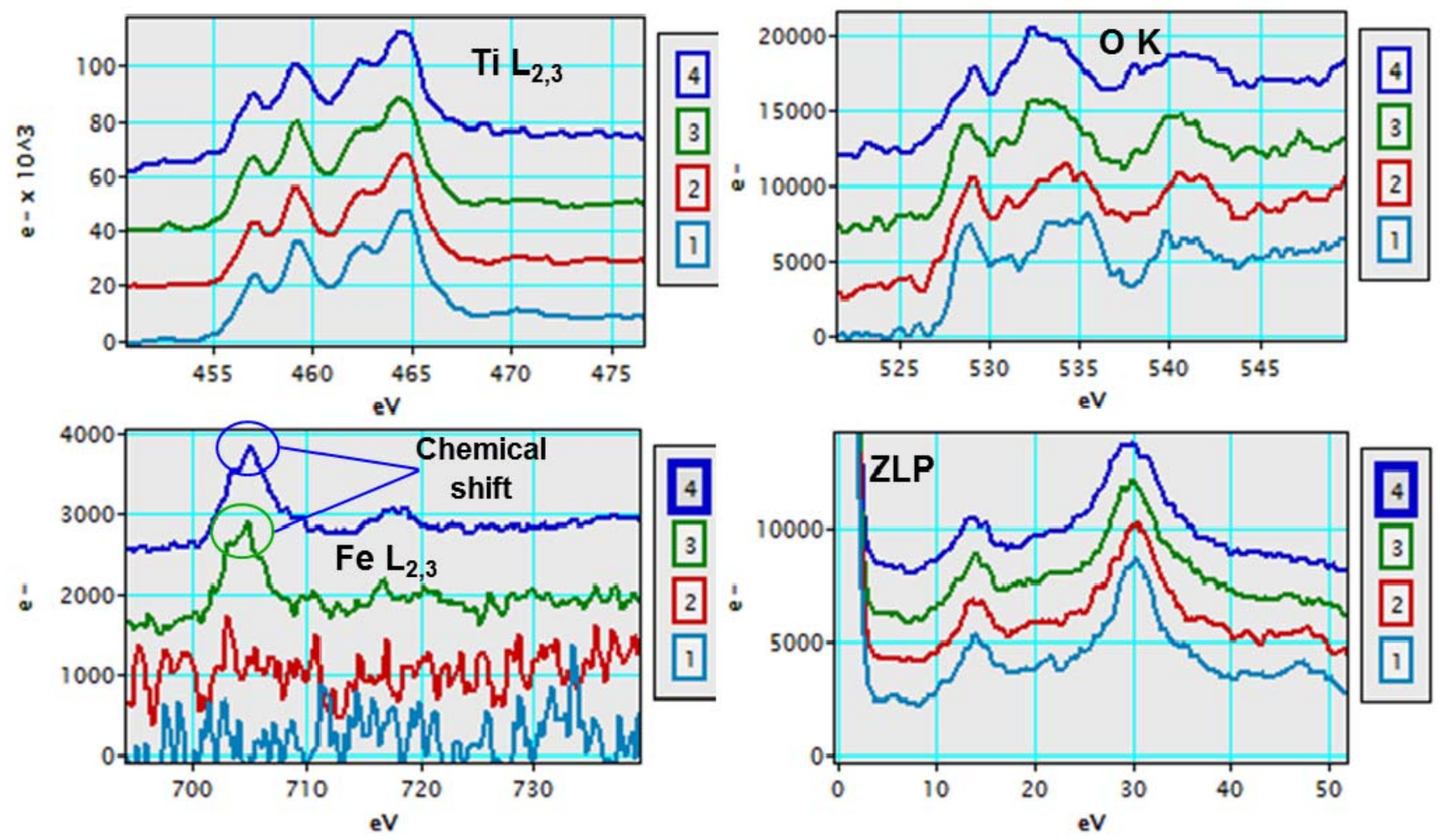

Figure 2: EELS spectra extracted from the selected region in Figure 1; a) $\mathrm{Ti} \mathrm{L}_{2,3}$-edges at 456eV; b) $\mathrm{O}$ K-edge at $532 \mathrm{eV} ; \mathrm{Fe} \mathrm{L}_{2,3}$-edges at $708 \mathrm{eV}$; d) Low-Loss region. 